Bundesgesundheitsbl - Gesundheitsforsch Gesundheitsschutz 2005 - 48:1038-1042 DOI 10.1007/s00103-005-1122-6 ๑) Springer Medizin Verlag 2005

A. Ammon · Robert Koch-Institut, Berlin

\title{
Strukturen der Überwachung und des Managements von Infektionskrankheiten in der EU
}

\section{Die EU-Netzwerke für übertragbare Krankheiten und das Europäische Zentrum für die Prävention und die Kontrolle von Krankheiten (ECDC)}

teriums für Gesundheit und Soziale Sicherung (BMGS), des Robert Koch-Instituts (RKI) und des Bundesrats repräsentiert (• Übersicht 1).

Zunächst wurde die Liste der auf EUEbene zu überwachenden Krankheiten festgelegt [2]. Als Kriterien für die Auswahl wurden folgende Punkte herangezogen:

1. Die Krankheit hat ein signifikantes Morbiditäts- oder Mortalitätspotenzial im europäischen Rahmen, insbesondere erfordert ihre Prävention einen gemeinschaftlichen Ansatz.

2. Der Austausch von Informationen über die Krankheit im Sinne einer Frühwarnung ist für andere MS sinnvoll.

3. Die Krankheit ist schwerwiegend, aber so selten, dass nur durch die $\mathrm{Zu}$ sammenführung der Daten eine Hypothesengenerierung möglich ist.

4. Für die Krankheit gibt es effektive Präventionsmaßnahmen.

5. Bei der Krankheit trägt ein Vergleich zwischen den MS zur Evaluation der nationalen oder der EU-Programme bei.

\section{Falldefinitionen}

Im Jahr 2002 wurden für die so festgelegten Krankheiten (Übersicht 2) dann auch die für Europa gültigen Falldefinitionen verabschiedet [3]. Sie sind seit Januar 2003 offiziell in Kraft. Bei der Entwicklung der Falldefinitionen für Deutschland wurde darauf geachtet, dass sie die Berichterstattung nach den EU-Falldefinitionen erlauben. Beim Blick auf die EU-Liste wird deutlich, dass die meisten, aber nicht al-

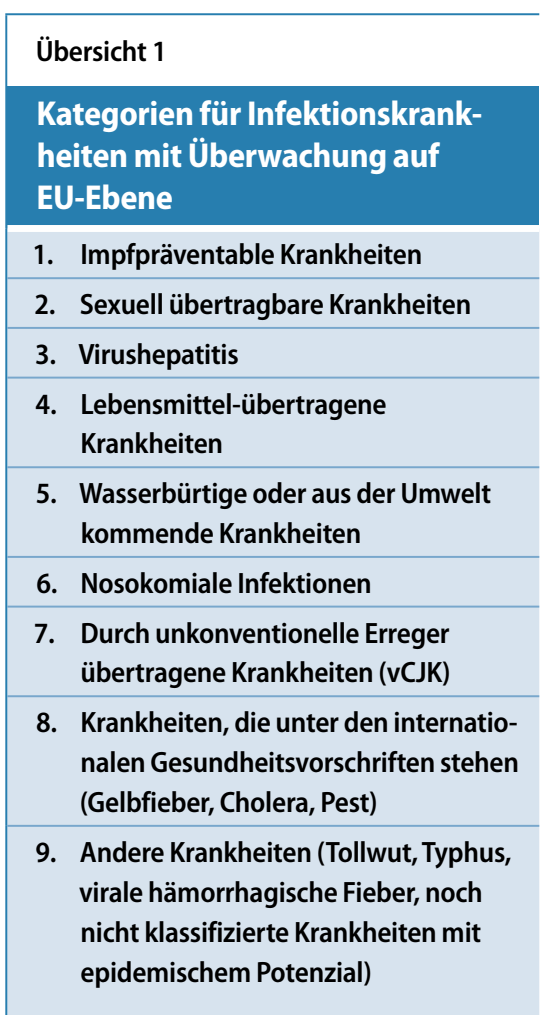


le der genannten Krankheiten in Deutschland meldepflichtig sind (insbesondere bei den impfpräventablen und sexuell übertragbaren Krankheiten). Die EU-Falldefinitionen gliedern sich in eine kurze Beschreibung des klinischen Bildes und in Laborkriterien. Daraus werden Definitionen für mögliche, wahrscheinliche und bestätigte Fälle entwickelt. Asymptomatische Fälle sollen nur dann mit einbezogen werden, wenn daraus therapeutische oder Public-Health-Konsequenzen resultieren. Nach dem Auftreten von SARS und den ersten Westnil-Virusinfektionen [4] wurden auch für diese Krankheiten Falldefinitionen im Rahmen des Netzwerks abgestimmt (• Übersicht 2).

\section{Frühwarnsystem}

Eine wichtige Rolle im EU-Netzwerk der übertragbaren Krankheiten kommt dem Frühwarnsystem Early Warning and Response System (EWRS) zu. Es besteht aus einem Web-basierten, Passwort-geschützten Meldesystem für wichtige Informationen, die schnell an alle MS gehen müssen. Wer in einem MS Zugang zu den Informationen des EWRS hat und zum Absetzen von Meldungen berechtigt ist, entscheiden jeweils die nationalen Gesundheitsbehörden. In Deutschland sind BMGS und RKI an die Informationen angeschlossen, das RKI ist beauftragt, falls erforderlich, Meldungen ins EWRS zu geben. Die Entscheidung 2119/98/EC verpflichtet die MS, Informationen über das Auftreten von auf EU-Ebene meldepflichtigen Infektionskrankheiten sowie die in diesem Zusammenhang getroffenen Maßnahmen an die anderen MS und die Kommission zu geben.

\section{$\rightarrow$ Zum schnellen Informations- austausch über Ausbrüche gibt es in der EU ein Web-basiertes Frühwarnsystem}

Beim Auftreten von neuen Infektionskrankheiten (wie es z. B. bei SARS oder bei der aviären Influenza geschehen ist), müssen MS ebenfalls sofort informieren. In solchen Situationen sind die Informationen für die anderen MS und die Kommission einmal aus Gründen der Vorwarnung wichtig, aber auch, um Maßnahmen

Bundesgesundheitsbl - Gesundheitsforsch - Gesundheitsschutz 2005 - 48:1038-1042 DOI 10.1007/s00103-005-1122-6

c) Springer Medizin Verlag 2005

\section{A. Ammon}

\section{Strukturen der Überwachung und des Managements von Infektionskrankheiten in der EU. Die EU-Netzwerke für übertragbare Krankheiten und das Europäische Zentrum für die Prävention und die Kontrolle von Krankheiten (ECDC)}

\section{Zusammenfassung}

Im Jahr 1998 wurde von EU-Parlament und Ministerrat die Entscheidung zur Etablierung eines EU-weiten Netzwerks zur Überwachung von Infektionskrankheiten getroffen. Bei der Entwicklung dieses Netzwerks legten Netzwerk-Komitee (die Vertreter der Mitgliedsstaaten) und Kommission zunächst die in der Surveillance zu erfassenden Krankheiten fest. Als nächster Schritt wurden Falldefinitionen für die EU-weite Überwachung geschaffen. Zum schnellen Informationsaustausch über schwerwiegende Krankheiten, Ausbrüche und die im Zusammenhang damit getroffenen Maßnahmen gibt es ein Web-basiertes Frühwarnsystem mit Passwort-geschütztem Zugang. Parallel dazu etablierten sich zur vertieften Erfassung wichtiger Erkrankungen krankheitsspezifische Surveillance-Netzwerke, die un- terschiedliche inhaltliche Schwerpunkte und Verfahrensweisen entwickelt haben. Ein Trainingsprogramm (EPIET) und das europäische Bulletin Eurosurveillance bilden krankheitsübergreifende Aspekte des Netzwerks. Im Rahmen der EU-Richtlinie zum Monitoring von Zoonosen werden Daten zu bestimmten zoonotischen Infektionskrankheiten aus dem Human-, Veterinärund Lebensmittelbereich auf EU-Ebene zusammenfassend bewertet. Im Mai 2005 ist die Koordination und Weiterentwicklung des Netzwerks auf das Europäische Zentrum für die Prävention und die Kontrolle von Krankheiten (ECDC) übergegangen.

\section{Schlüsselwörter}

EU · Falldefinitionen · SurveillanceNetzwerke · Zoonosen-Richtlinie · ECDC

\section{Surveillance for infectious diseases in the European Union. The EU surveillance networks and the European Centre for Disease Prevention und Control (ECDC)}

\section{Abstract}

In 1998, the European Parliament and Council published the Decision on setting up a network for the epidemiological surveillance of communicable diseases in the Community. As a first step, the Network Committee (representatives of the Member States) and the Commission determined the diseases for European surveillance. Subsequently, case definitions were developed for these diseases. For rapid information exchange among Member States on serious diseases, outbreaks and the control measures taken, a web-based, password protected early warning system was established. In parallel disease-specific networks were developed for enhanced surveillance of impor- tant diseases. A training programme (EPIET) and a European bulletin (Eurosurveillance) complete the network. The directive on the monitoring of zoonoses constitutes the basis for the joint analysis and evaluation of data on human illnesses, animals and foods. Starting in May 2005, the coordination and further development of the Community Network will be carried out by the European Centre for Disease Prevention and Control (ECDC).

\section{Keywords}

EU - Case definitions · Surveillance networks . Zoonoses directive $\cdot$ ECDC 
Übersicht 2

Infektionskrankheiten, für die eine EU-weite Überwachung vorgesehen ist

1. Impfpräventable Krankheiten Diphtherie, Haemophilus influenzae B (invasive Erkrankungen), Influenza, Masern, Mumps, Pertussis, Poliomyelitis, Röteln, Tetanus

2. Sexuell übertragene Krankheiten Chlamydien, Gonorrhoe, HIV, AIDS, Syphilis

3. Virushepatitis Hepatitis A, B, C

4. Lebensmittel- und Wasser-übertragene Krankheiten oder aus der Umwelt kommende Krankheiten Botulismus, Campylobacteriose, Kryptosporidiose, Giardiasis, EHEC-Infektionen, Leptospirose, Listeriose, Milzbrand, Salmonellose, Shigellose, Toxoplasmose, Typhus/ Paratyphus, Trichinellose, Yersiniose

5. Durch unkonventionelle Erreger übertragene Krankheiten Variante Creutzfeldt-Jacob-Krankheit

6. Aerogen übertragene Krankheiten Legionellose, MeningokokkenErkrankungen, invasive Pneumokokken-Erkrankungen, Tuberkulose

7. Zoonosen (zusätzlich zu 4.) Brucellose, Echinokokkose, Q-Fieber, Tollwut, Tularämie

8. Importierte Krankheiten Cholera, Pest, Malaria, virale hämorrhagische Fieber (z. B. Lassa, Krim-Kongo, Gelbfieber)

9. Nosokomiale Infektionen

10. Antibiotikaresistenzen

ggf. abstimmen zu können. Da die Regelungen in der Entscheidung 2119/98/EC sehr allgemein gehalten sind, sodass bei deren Einhaltung das System schnell mit einer Flut (in der Regel) weniger nützlicher Meldungen überschwemmt würde, wurde versucht, die zu berichtenden Ereignisse zu kategorisieren und die Dringlichkeitsstufe zu definieren [5]. Es sollen Ausbrüche berichtet werden, die bereits mehr als einen MS involvieren, oder zum Berichtszeitpunkt zwar noch auf einen MS beschränkt sind, aber das Potenzial haben, sich auf andere MS oder die gesamte EU

Übersicht 3

EU-Surveillance-Netzwerke

1. Übergreifendes Netzwerk BSN (Basic Surveillance Network)

2. Sexuell/durch Blut übertragbare Erkrankungen Euro-HIV (European Centre for the Epidemiological Monitoring of AIDS), ESSTI (European Surveillance of Sexually Transmitted Infections)

3. Impfpräventable Erkrankungen ESEN (European Seroepidemiology Network), ELWGD (European Laboratory Working Group on Diphtheria), EUVAC.NET (Surveillance Community Network for Vaccine Preventable Infectious Diseases), EU IBIS (European Union Invasive Bacterial Infections Surveillance)

4. Zoonosen/Lebensmittel-bedingte Erkrankungen Enternet (International Surveillance Network for the Enteric Infections Salmonella and VTEC)

5. Respiratorische Erkrankungen Euro-TB (European Surveillance of Tuberculosis), EISS (European Influenza Surveillance Scheme), EWGLINet (European Working Group for Legionella Infections)

6. Antibiotikaresistenz/Nosokomiale Infektionen

EARSS (European Antimicrobial Resistance Surveillance System), ESAC (European Surveillance of Antimicrobial Consumption), HELICS (Hospitals in Europe Link for Infection Control through Surveillance)

7. Andere

ENIVD (European Network for Imported Viral Diseases)

auszubreiten. Insbesondere muss dann informiert werden, wenn zur Bekämpfung ein EU-weit koordinierter Ansatz notwendig ist. In einer Reihe von Netzwerk-internen Arbeitsdokumenten wurden weitere Konkretisierungen getroffen, die aber derzeit noch in Erprobung sind. Während des SARS-Ausbruchs im Jahr 2003 hatte dieses System seine erste Bewährungsprobe. Alle MS waren sich einig, dass damit ein guter Austausch von Informationen, insbesondere über geplante Maßnahmen, möglich war, dass jedoch die Informationsflut mit 10-20 Meldungen pro Tag (neben den anderen eingehenden Informationen) $\mathrm{z}$. T. nicht mehr verarbeitet werden konnte. Inzwischen gibt es auch in allen MS und der Kommission eine 24-Stunden/7-Tage-Kontaktmöglichkeit, sodass in Notfällen auch Ansprechpartner vorhanden sind.

\section{Krankheitsspezifische Netzwerke - Surveillance-Netzwerke}

Zur Überwachung der verschiedenen Infektionskrankheiten werden krankheitsspezifische Netzwerke gefördert (Übersicht 3). Zum Teil waren diese schon vor der Entscheidung 2119/98/EC entstanden (z. B. Enter-net, Euro-HIV). Die spezifischen Netzwerke haben - angepasst an die betreffende Krankheit - jeweils ein eigenes Arbeitsprogramm entwickelt. In der Regel wurde versucht, zur jeweiligen Krankheit die nationalen Experten aus Epidemiologie und Labor (je einen pro Disziplin pro MS) aus möglichst vielen MS zusammenzubringen, um durch die Zusammenführung von Daten einen sog. „Europäischen Mehrwert“ zu erzielen. Dieser kann sich zum einen in einer Standardisierung der verwendeten epidemiologischen Erhebungsmethoden oder der angewandten Labormethoden zeigen, zum anderen aber auch in der Identifikation von Länder übergreifenden Ausbrüchen liegen, die andernfalls nicht oder später entdeckt worden wären. Dies ist im Besonderen im Rahmen von Enter-net, dem Netzwerk für die (vor allem) Lebensmittel-bedingten Infektionen durch Salmonellen, Shigatoxin produzierende E. coli (STEC) und Campylobacter bereits mehrmals der Fall gewesen $[6,7,8]$ (• Übersicht 3).

\section{Zur vertieften Erfassung wichtiger Infektionskrankheiten haben sich in der EU krankheits- spezifische Surveillance- Netzwerke entwickelt}

Da die Netzwerke als Projekte unabhängig voneinander beantragt wurden, haben sich innerhalb dieser zwangsläufig uneinheitliche Verfahrensweisen entwickelt (hinsichtlich der inhaltlichen Schwerpunkte, der Verfahrensweisen zur Auswertung der Daten sowie zur Information der Netzwerkpartner und der weiteren Fachöffentlichkeit). Im Rahmen des Europäischen 
Zentrums für die Prävention und die Bekämpfung von Krankheiten (ECDC, s. unten) werden diese Netzwerke daher evaluiert und die Verfahrensweise der dann DSN (Dedicated Surveillance Networks) genannten Netzwerke vereinheitlicht werden [9].

Als übergreifendes Netzwerk für die Sammlung von epidemiologischen Basisdaten wurde das sog. BSN (Basic Surveillance Network) entwickelt. Hier werden von einem (zunehmend größeren) Teil der MS regelmäßig (monatlich) Basisinformationen (Land, Meldewoche, Alter, Geschlecht, zugrunde liegende Krankheit, Kategorie der Falldefinition) zu EU-weit meldepflichtigen Krankheiten erhoben. Da dieses Verfahren gut funktioniert, könnte dieses Netzwerk als Grundstein für die EU-Surveillance ausgebaut werden. Im Rahmen der DSN könnten dann Zusatzinformationen (Symptome, Impfstatus, Laborinformationen einschließlich der Typisierungsergebnisse) erhoben werden.

\section{Übergreifende Projekte}

Zwei krankheitsübergreifende Projekte, die das Netzwerk ergänzen, sollen hier noch erwähnt werden: das Trainingsprogramm EPIET (European Programme for Intervention Epidemiology Training) und Eurosurveillance, das europäische Bulletin (monatlich, wöchentlich und als E-Alert).

EPIET. Das Trainingsprogramm EPIET hat in den vergangenen 10 Jahren nicht nur für die Ausbildung von Infektionsepidemiologen mit europäischer Orientierung gesorgt, sondern durch die Ausrichtung von Kursen und die Betreuung der Trainees in den verschiedenen Gastinstituten einen großen Beitrag zum Aufbau und zur Vertrauensbildung im Netzwerk geleistet (Näheres s. Beitrag von V. Bremer in diesem Heft).

Eurosurveillance. Mithilfe des europäischen Bulletins Eurosurveillance sollen Informationen zu Präventionsmaßnahmen innerhalb des Netzwerks schnell und zuverlässig verbreitet werden. Sowohl die Artikel in der wöchentlichen als auch die in der monatlichen Ausgabe werden vor der Veröffentlichung von Experten begutachtet (peer-review), die Monatsausgabe ist auch in der Datenbank Medline/
Index Medicus gelistet. Zur Kommunikation von dringenden Informationen wurde Eurosurveillance E-Alert eingeführt. Hier werden dringende Nachrichten per E-Mail an alle Bezieher weitergegeben.

\section{Grenzland zum \\ Veterinär-/Lebensmittelbereich: die Zoonosenrichtlinie}

Im November 2003 wurde die neue Richtlinie zum Monitoring der Zoonosen von der EU (Parlament und Ministerrat) verabschiedet [10]. Damit wurde die Richtlinie aus dem Jahr 1992 abgelöst. Nach der neuen Regelung, die seit dem 12.6.2004 in allen EUMitgliedsstaaten gilt, müssen folgende Zoonosen in das regelmäßige Monitoring eingeschlossen werden: Brucellose, Campylobacteriose, Echinokokkose, Listeriose, Salmonellose, Trichinellose, Tuberkulose durch Mycobacterium bovis und Erkrankungen durch Verotoxin bildende (Shigatoxin bildende) Escherichia coli. Für diese Erreger müssen sowohl die Zahlen aus dem Veterinärbereich als auch humane Erkrankungsdaten gesammelt werden. Zudem müssen Daten zur Antibiotikaresistenz mindestens von Salmonellen und Campylobacter bei Rindern, Schweinen, Geflügel und von diesen Tieren stammenden Lebensmitteln mitgeteilt werden. Als dritter Bereich, zu dem Daten berichtet werden müssen, sind - ganz neu in dieser Richtlinie - Daten von Lebensmittel-bedingten Ausbrüchen zu melden. Diese Ausbruchsberichte müssen folgende Angaben enthalten:

- Gesamtzahl der Ausbrüche innerhalb eines Jahres,

- Zahl der menschlichen Erkrankungsund Todesfälle in diesen Ausbrüchen,

- verursachender Erreger, einschließlich, wenn vorhanden, Serotyp oder weitere Charakterisierung,

- Lebensmittel, das/die mit dem Ausbruch in Zusammenhang gebracht wird/werden,

- Art des Ortes, wo das implizierte Lebensmittel hergestellt/gekauft/erworben/verzehrt wurde,

- beitragende Faktoren, z. B. Hygienemängel in der Herstellung.

Den EU-Zoonosenbericht (d. h. die Zusammenfassung der Daten auf EU-Ebe- ne) wird die European Food Safety Authority (EFSA) erstellen. Zu diesem Zweck müssen die MS die Veterinärdaten direkt an die EFSA leiten, die Humandaten werden vom Europäischen Zentrum für Prävention und Kontrolle von Krankheiten (ECDC) zur EFSA übermittelt. Der entsprechende Verfahrensablauf ist derzeit in der Diskussion. Um die Informationen berichten zu können, ist es unabdingbar, dass bei Lebensmittel-bedingten Ausbrüchen die Gesundheits- und Veterinärbehörden vor Ort eng zusammenarbeiten. In Deutschland wird versucht, diese Zusammenarbeit durch ZEVALI (zentrale Erfassung von Ausbrüchen Lebensmittel-bedingter Infektionen) zu fördern. Die EFSA hat derzeit eine Ausschreibung für ein System zur Erfassung der Lebensmittel-bedingten Ausbrüche auf EU-Ebene laufen.

Auch wenn im Moment noch nicht alle Verfahren der künftigen Zoonosenberichterstattung festgelegt sind, zeigen die Diskussionen aber bereits den Wert des Netzwerks: Die Ansprechpartner sind bekannt, und somit können Fragen und Probleme diskutiert und Lösungen gefunden werden.

\section{Das Europäische Zentrum zur Prävention und Bekämpfung von Krankheiten}

Im April 2004 wurde durch das Europäische Parlament die Errichtung des ECDC verabschiedet [11]. Es hat am 20. Mai 2005 seine Arbeit aufgenommen. Obwohl die Notwendigkeit der Einrichtung einer Koordinationsstelle für das in den letzten Jahren aufgebaute und gewachsene Netzwerk zur Überwachung von Infektionskrankheiten in der EU von den meisten MS anerkannt wird, waren der Entscheidung im Parlament heftige Diskussionen zwischen den MS und der Kommission vorausgegangen, in denen es um die Kompetenzen, aber auch die Ausstattung des ECDC ging. Mit der jetzigen Regelung wird festgelegt, dass das Europäische Zentrum keinerlei regulative Macht hat, sondern die folgenden Bereiche koordinieren soll:

\section{Epidemiologische Surveillance und} Labornetzwerk.

Das ECDC soll die Weiterentwicklung des begonnenen Netzwerks der europä- 
ischen Infektionsüberwachung übernehmen. Eine Datenbank für die EU-weiten Surveillancedaten soll dort etabliert werden. Die Verfahrensweisen der bisher weitgehend voneinander unabhängigen Surveillance-Netzwerke für die verschiedenen Krankheiten sollen vereinheitlicht und vom Zentrum koordiniert werden. Da das Zentrum keine eigenen Laboratorien haben wird, soll ein Netzwerk aus Referenzlaboren geschaffen werden. Hierzu sind im Rahmen der bisherigen krankheitsspezifischen Netzwerke bereits Kooperationen zwischen Laboratorien in den MS etabliert worden, die jetzt weiterentwickelt und auf zusätzliche Erreger ausgedehnt werden müssen.

\section{Das Early Warning and Response} System

Hier ist die Aufgabe, das bisherige System des schnellen Informationsaustauschs nahtlos zu übernehmen und weiterzuentwickeln. Die Verantwortlichkeit für zu treffende Maßnahmen bleibt jedoch bei den Gesundheitsbehörden der MS.

\section{Technische Unterstützung der MS}

Falls angefordert, könnte das Zentrum ein EU-Team zur Unterstützung bei Ausbrüchen und Krisen innerhalb, aber auch außerhalb der EU zusammenstellen und entsenden.

\section{Kommunikation}

Das ECDC soll die ihm berichteten Daten analysieren, interpretieren und die Ergebnisse den Entscheidungsträgern in den MS, dem EU-Parlament, der Kommission und anderen Organisationen zeitnah und in geeigneter Form zur Verfügung stellen.

\section{Erstellung von wissenschaftlichen}

\section{Gutachten}

Wissenschaftliche Fragestellungen aus allen Bereichen der Infektionskrankheiten sollen vom ECDC bearbeitet werden, sei es auf Anfrage der Kommission, des EU-Parlaments oder aufgrund eigener Entscheidung. Falls die Eigenexpertise des Zentrums zur Bearbeitung nicht ausreicht, können bei Bedarf Ad-Hoc-Panels von Experten aus den MS zu spezifischen Fragen einberufen werden, die das Zentrum beraten.

Wie bereits mehrfach betont $[12,13,14]$, werden an das ECDC hohe Erwartungen seitens der Politik, aber auch der Fachöffentlichkeit gestellt. Diesen entspricht aber das dem Zentrum vom Parlament zugeteil- te Budget und auch die Personalausstattung nicht. Daher wird es zur Erfüllung dieser Ansprüche der temporären Entsendung von Wissenschaftlern aus den MS bedürfen.

\section{Abschließende Betrachtung}

Die zunehmende Bedeutung des Bereichs Public Health und hier insbesondere der Infektionskrankheiten im Rahmen der EU spiegelt die Tatsache wider, dass sich bei der gegebenen Mobilität von Menschen, Tieren und Lebensmitteln auch die entsprechenden Erreger schnell über Landesgrenzen hinweg ausbreiten können. Insofern ist der Gedanke an ein Länder übergreifendes Netzwerk zur Überwachung von Infektionskrankheiten nahe liegend. Das RKI sieht sich hier als Mittler zwischen dem nationalen und den internationalen Netzwerken.

Die Überwachung von Infektionskrankheiten kann auf EU-Ebene nur dann erfolgreich sein, wenn auch die einzelnen nationalen Systeme gut „aufgestellt" sind, d. h., wenn die nationale Infektionsepidemiologie in der Lage ist, im erforderlichen Ausmaß qualitätsgesicherte Daten zeitnah zur Verfügung zu stellen, und sie die sich daraus ergebenden Fragestellungen oder Konsequenzen auch mittels infektionsepidemiologischer Forschung bearbeiten kann. Hier hat Deutschland in den letzten 10 Jahren viel verlorenen Boden gut gemacht. Es gilt jetzt, die etablierten Strukturen weiterzuentwickeln, um so den Anschluss an den Stand der Wissenschaft zu halten. Dies kann jedoch nur gelingen, wenn die dafür erforderlichen Ressourcen vor Ort in den Gesundheitsämtern, auf der Ebene der Länder, aber auch beim Bund zur Verfügung stehen.

\section{Korrespondierender Autor \\ Dr. A. Ammon}

Abteilung für Infektionsepidemiologie,

Robert Koch-Institut,

Postfach 650261, 13302 Berlin

E-Mail:AmmonA@rki.de

\section{Literatur}

1. Decision No 2119/98/EC of the European Parliament and of the Council setting up a network for the epidemiological surveillance and control of communicable diseases in the Community. OJ L268, 3.10.1998, p.1
2. Commission Decision 2000/96/EC on the communicable diseases to be progressively covered by the Community network under Decision No. 2119/98/EC of the European Parliament and of the Council. OJ L28, 3.2.2000, p.50

3. Commission Decision 2002/253/EC laying down case definitions for reporting communicable diseases to the Community network under Decision No. 2119/98/EC of the European Parliament and of the Council. OJ L 86, 3.4.2002, p.44

4. Connell J, McKeown P, Garvey P et al. (2004) Two linked cases of West Nile virus (WNV) acquired by Irish tourists in the Algarve, Portugal. Eurosurveill Weekly 8:5.8.2004

5. Commission Decision 2000/57/EC on the early warning and response system for the prevention and control of communicable diseases under Decision No. 2119/98/EC of the European Parliament and of the Council. OJ L 21, 26.1.2000, p.32

6. Werber D, Dreesman J, Feil F et al. (2005) International outbreak of Salmonella Oranienburg due to German Chocolate. BMC Infect Dis 5:7

7. Crook PD, Aguilera JF, Threllfall EJ et al. (2003) A European outbreak of Salmonella enterica serotype Typhimurium definitve phage type 204b in 2000. Clin Microbiol Infect 9:839-845

8. Lieftucht $A$, Reacher $M$, the international outbreak control team (1999) Case control study of Salmonella paratyphi B infection associated with travel to Alanya, Turkey: update. Eurosurveill Weekly 3:28.10.1999

9. Commission Decision 2003/542/EC amending Decision 2000/96/EC as regards the operation of dedicated surveillance networks. OJ L55, 24.7.2003, p.55

10. Directive 2003/99/EC of the European Parliament and of the Council on the monitoring of zoonoses and zoonotic agents, amending Council Decision 90/424/EC and repealing Council Directive 92/117/ EEC. OJ L 325, 12.12.2003, p.31

11. Regulation (EC) No. $851 / 2004$ of the European Parliament and of the Council of 21.4.2004 establishing a European centre for disease prevention and control. OJ L142, 30.4.2004, p.1

12. Norrby SR (2004) Alert to a European epidemic. Nature 431:507-508

13. Wigzell H (2005) A European CDC? Science 307:1691

14. Vermij $P$ (2005) Money, manpower missing from Europe's new agency. Nat Med 11:358 